\title{
Corela
}

Cognition, représentation, langage

HS-7 | 2010

Espace, Préposition, Cognition

\section{Le localisme à l'épreuve du verbe aller}

\section{Bernard Victorri}

\section{OpenEdition}

\section{Journals}

Édition électronique

URL : http://journals.openedition.org/corela/1009

DOI : $10.4000 /$ corela. 1009

ISSN : 1638-573X

\section{Éditeur}

Cercle linguistique du Centre et de l'Ouest - CerLICO

\section{Référence électronique}

Bernard Victorri, « Le localisme à l'épreuve du verbe aller », Corela [En ligne], HS-7 | 2010, mis en ligne le 31 mai 2010, consulté le 02 mai 2019. URL : http://journals.openedition.org/corela/1009 ; DOI : 10.4000/corela.1009

Ce document a été généré automatiquement le 2 mai 2019.

\section{(c) (i) (2)(2)}

Corela - cognition, représentation, langage est mis à disposition selon les termes de la licence Creative Commons Attribution - Pas d'Utilisation Commerciale - Partage dans les Mêmes Conditions 4.0 International. 


\section{Le localisme à l'épreuve du verbe aller}

\section{Bernard Victorri}

\section{Introduction}

1 Dans l'un des tout derniers articles qu'il a publiés, intitulé Le verbe ALLER L'affranchissement du contexte d'énonciation immédiat (Vandeloise 2007), Claude Vandeloise s'attaque de nouveau à la question de la pertinence de la thèse localiste, que Lyons résumait ainsi ${ }^{1}$ : «les expressions spatiales sont plus fondamentales, grammaticalement et lexicalement, que diverses espèces d'expressions non spatiales » (Lyons 1980, p. 338). A cette question, qu'il n'a cessé d'approfondir tout au long de ses recherches, Vandeloise a apporté des réponses de plus en plus nuancées. C'est ainsi qu'il propose deux caractérisations du verbe aller, ouplus précisément d'un certain nombre d'emplois de ce verbe, car, selon son habitude, il délimite précisément les emplois qu'il fait entrer dans son champ d'étude. L'une de ces caractérisations peut être traitée de localiste, à condition d'accepter, comme il le défendait depuis longtemps, de considérer l'espace dans lequel nous évoluons comme plus riche qu'un simple espace géométrique, en le dotant des propriétés dynamiques, fonctionnelles et intentionnelles que lui confère notre expérience de vie quotidienne. L'autre caractérisation est plus originale dans son travail : elle repose sur la notion de contexte d'énonciation, ce qui n'est pas très courant dans son dispositif théorique, et elle est accompagnée par une réflexion sur l'origine et l'évolution du langage, thèmes qu'il n'avait, à ma connaissance, jamais abordés jusque là.

2 Déjà dans un article publié l'année précédente (Vandeloise 2006), il avait répondu de manière doubleà la question : Are there spatial prepositions? La première réponse, positive, relevait aussi d'un "localisme enrichi", tel qu'on vient de le définir, et la deuxième, négative, était elle aussi plus abstraite, mais plus classique dans l'approche de Vandeloise, puisqu'elle s'appuyait ce qu'il appelle des primitives complexes, du type contenant/contenu et charge/support. Notons aussi que dans les deux articles, les caractérisations localistes 
sont mises en contribution pour rendre compte de l'évolution diachronique des langues et leur acquisition par les enfants.

$3 \quad$ Ainsi le double point de vue de Vandeloise sur le verbe aller n'est pas un accident. On peut penser que cela fait partie au contraire d'un cadre théorique général qu'il était progressivement en train de mettre en place, et que l'on pourrait résumer ainsi :

- Le premier niveau de description correspondrait à la connaissance que les locuteurs naïfs ont de leur langue, et jouerait un rôle décisif dans le processus d'acquisition de la langue par l'enfant et l'évolution diachronique des langues. L'hypothèse localiste, convenablement enrichie, serait vérifiée pour ce niveau.

- Le deuxième niveau de description, beaucoup plus abstrait, faisant appel à des primitives complexes ainsi qu'à des propriétés énonciatives, correspondrait mieux à l'utilisation que font les adultes de leur langue, et notamment à l'exploitation que font les écrivains de sa richesse. A ce niveau la primauté du spatial serait totalement perdue, et donc l'hypothèse localiste invalidée.

- Ces deux niveaux seraient complémentaires et non contradictoires. Un principe général d'extension des sens d'un mot, que Vandeloise appelle son impulsion logique permettrait de comprendre l'ensemble de la distribution des sens d'un mot en articulant les deux niveaux de description. C'est aussi grâce à cette articulation que pourraient être décrites les grandes étapes de la genèse du langage, depuis les premières interactions linguistiques des ancêtres de l'Homme jusqu'à nos langues actuelles.

C'est en ayant en tête ce cadre théorique général, que Claude Vandeloise n'aura malheureusement pas eu le loisir d'expliciter davantage, que je vais examiner en détail son analyse du verbe aller. Mes commentaires critiques n'auront d'autre ambition que de faire écho aux discussions passionnées que nous avions engagées depuis une dizaine d'années, et qui, à mon grand regret, ont été brutalement et cruellement interrompues.

\section{Une classification des emplois du verbe aller}

5 Vandeloise commence par classer les emplois de aller suivant leur construction. Remarquant que ce verbe est obligatoirement suivi d'un complément, il propose une séparation en deux classes, basée sur la nature de ce complément :

- Avec un complément adverbial (aller vite, aller à pied), aller est un verbe de manière de déplacement. Par extension, entrent aussi dans cette classe les emplois hors du domaine spatial qui obéissent à la même construction (Louis va bien).

- Avec un complément de lieu introduit par une préposition spatiale (aller à Prague) ou un verbe à l'infinitif (Yvonne va manger, dans le sens où Yvonne se déplace pour manger), aller est un verbe de déplacement. Là aussi, par extension, on fait entrer dans cette classe les emplois de futur proche (Yvonne va manger, au sens où Yvonne est sur le point de manger).

Cette classification dichotomique peut susciter un certain nombre d'objections, d'importance plus ou moins grande, qu'il nous faut discuter avant d'aller plus loin.

On peut d'abord contester le fait que le verbe aller réclame toujours un complément, comme on pourra le constater avec (entre autres...) les exemples suivants:

Ils vont et viennent

Va, cours, vole et nous venge!

Laissez-le aller, il n'a rien fait !

Est-ce que ça va ? Ça peut aller. Rien ne va plus !, etc.

Allez ! Allons bon! Va donc !, etc. 
facilement à une classe ou a l'autre sur la base de leur proximité sémantique avec des facilement à une classe ou à l'autre sur la base de leur proximité sémantique avec des emplois déjà classés (comme Ils vont de long en large pour le premier exemple ou Va nous venger pour le vers de Corneille), mais on a perdu le critère formel que nous proposait Vandeloise. De même, ce critère est inutilisable dans les cas, encore plus nombreux, où aller est construit avec les deux types de complément à la fois (aller à pied à Prague). Quel complément privilégier dans ce cas ? Enfin, il est aussi assez difficile de trancher pour un certain nombre de cas intermédiaires, où l'on a du mal à choisir entre "expression adverbiale » et " complément de lieu » pour reprendre les termes exacts de Vandeloise. Ainsi, s'il est facile de choisir la bonne classe pour aller en bateau et aller en Espagne, c'est moins évident pour aller en voyage, aller en mer, aller en vacances ou encore aller à travers champs.

9 Le problème se pose avec d'autant plus d'acuité que Vandeloise ne semble faire cette classification dichotomique des emplois que pour le verbe aller, les autres verbes qu'il cite ne relevant que de l'une des deux classes: verbes de déplacement pour partir, passer, arriver, entrer, sortir et verbes de manière de déplacement pour marcher, voler, et aussi d'ailleurs se déplacer. Or la plupart de ces verbes acceptent aussi des compléments des deux classes : à côté de partir en Espagne et de passer par Bordeaux, on peut avoir partir vite et passer en voiture, et, pour ce qui est des verbes de manière de déplacement, marcher vite et se déplacer en voiture n'empêchent pas marcher de la maison au bureau ou se déplacer sur les lieux du crime.

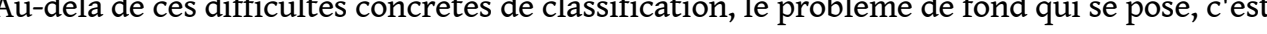
la pertinence même de cette distinction entre déplacement et manière de déplacement. Elle provient sans doute de la fameuse distinction typologique introduite par Talmy (1985) entre langues à cadrage verbal et langues à cadrage satellitaire (verb-framed versus satellite-framed languages), qu'illustre, par exemple, le contraste entre Il a traversé la rivière à la nage et He swam through the river. (cf. notamment Slobin 2003 pour l'usage qu'en ont fait les psycholinguistes). Mais comme l'ont bien montré Pierre Cadiot et ses collègues (Cadiot et al. 2006), même si cette distinction typologique avérée peut se révéler utile comme heuristique, son intérêt théorique est moins évident. Notamment, ils font remarquer qu'une langue à cadrage verbal comme le français utilise très naturellement des constructions à cadrage satellitaire (Il vole de branche en branche, il a dansé jusque chez lui, il a sauté par-dessus la barrière), et, de plus, que dans la plupart des constructions dites à cadrage verbal, ce n'est pas le verbe seul qui spécifie le déplacement mais l'interaction entre le verbe et la préposition du satellite, comme le montrent par exemple les différences subtiles mais sensibles de configuration des mouvements exprimés par partir suivi de diverses prépositions : partir à Paris, partir pour Paris, partir sur Paris, partir vers Paris. On peut donc à bon droit trouver problématique la transposition de cette distinction typologique, incontestable mais sans grande portée théorique, au classement des verbes au sein du lexique d'une même langue, et plus encore, au classement des différents sens d'un même verbe.

11 Un autre problème qu'il nous faut soulever concerne les extensions de cette classification à des sens non spatiaux, qui conduisent Vandeloise à considérer que les emplois de type aller bien se rattachent aux emplois de verbe de manière de déplacement et que le sens de futur proche se rattache aux emplois de verbe de déplacement. On peut noter tout d'abord que Vandeloise est loin d'épuiser ainsi tous les sens du verbe aller, notamment ceux qu'il prend dans les exemples suivants : 
Ce chapeau lui va bien

Il va sur ses quarante ans

Il n'aurait pas dû se laisser aller

Il va jusqu'à envisager de tout plaquer construction : un complément adverbial pour la manière de déplacement, et un verbe à l'infinitif pour le déplacement. Il faut aussi remarquer qu'il n'accorde pas la même attention aux deux cas : alors qu'il discute de manière détaillée l'emploi de futur proche dans la suite de l'article, il écarte assez vite du champ de son étude les usages de verbe de manière de déplacement et leurs extensions, en se contentant de noter qu'il existe une relation métaphorique générale, à la Lakoff (Lakoff et Jonhson, 1980), entre la manière de se déplacer et la manière de vivre ou de fonctionner (cf. ma montre marche bien).

En fait, l'objectif de Vandeloise dans cette introduction est très ciblé : il s'agit simplement de motiver son choix de restreindre son étude à certains emplois du verbe aller. Ce choix est un choix méthodologique, et, en tant que tel, il n'est pas critiquable : la complexité du verbe aller est telle qu'il est effectivement très raisonnable de ne pas aborder tous les problèmes à la fois, et l'une des méthodes les plus simples de procéder, c'est effectivement de se limiter, dans un premier temps, à une partie de ses emplois.

Mais la contrepartie de ce choix méthodologique, c'est bien entendu de s'assurer par la suite que les résultats obtenus sur les emplois étudiés sont bien compatibles, en un sens ou un autre, avec les emplois écartés. C'est la raison pour laquelle il était nécessaire de discuter un peu longuement le tableau d'ensemble des emplois de aller que brosse Vandeloise dans son introduction. En effet, nous pouvons retenir de cette discussion les deux impératifs suivants :

- Toute caractérisation de aller comme verbe de déplacement doit pouvoir être facilement étendue aux emplois dits de verbe de manière de déplacement, puisque, on l'a vu, il ne saurait y avoir de coupure franche entre les deux séries d'emplois.

- Toute caractérisation de aller dans le domaine spatialdoit pouvoir être étendue, d'une manière ou d'une autre, aux emplois non spatiaux de aller, et pas seulement à ceux qui partagent une construction commune avec des emplois spatiaux, sous peine d'être contraint à accepter la thèse éminemment contestable de l'homonymie : choix que Vandeloise luimême récuse pour l'un des emplois de aller qui pourtant s'y prête le plus, à savoir l'emploi grammatical de marque de futur proche.

C'est à la lumière de ces contraintes que nous allons maintenant examiner les propositions de Vandeloise pour caractériser les emplois de aller comme verbe de déplacement.

\section{La caractérisation localiste}

Vandeloise propose la règle suivante pour caractériser le sens de aller suivi d'un complément de lieu :

$\mathrm{R}_{1}$ : $x$ va Prep $y$ si $x$ change de lieu au moment de l'énonciation et si le lieu final anticipé est Prep $y$.

18 Cette règle est une reformulation de la règle qu'il avait proposée vingt ans auparavant (Vandeloise 1987) : x va Prep y si $x$ est en déplacement (anticipé) au moment de l'énonciation et si le terme anticipé du déplacement est Prep y. 

la fonction exacte du complément spatial. La principale modification concerne la notion de lieu qui entre en force dans la nouvelle formulation. Ce n'est pas un détail, mais au contraire un véritable changement d'approche. En effet, Vandeloise donne une définition précise de ce qu'il appelle un lieu, à savoir « une position dans l'espace chargée d'une valeur aux yeux des participants au discours» (p. 348). Un peu plus loin, Vandeloise ajoute: "Puisqu'un lieu est une position chargée d'une valeur fonctionnelle, leur existence est entièrement subjective ». Il s'agit donc bien d'une notion fonctionnelle, voire énonciative, puisque les lieux sont construits dans et par le discours. Les exemples que donne Vandeloise à ce propos ne laissent aucun doute. Comparant les phrases Yvan marche dans la maison et Yvan va (de la cuisine) au salon, il explique qu'elles peuvent toutes deux décrire le même trajet objectif d'Yvan, mais que la première le présente comme une marche sans but, attribuant à toutes les positions occupées la même valeur, alors que la deuxième le présente comme un déplacement motivé, attribuant au salon une valeur particulière.

Il faut donc souligner le chemin théorique parcouru par Vandeloise en vingt ans : alors que ses premiers travaux, et notamment son premier livre sur l'espace en français (Vandeloise 1986) présentent un point de vue localiste "objectiviste", au sens où les expressions spatiales servent à décrire des situations spatiales objectives dans le monde, il développe ici une approche qui reste, certes, localiste, mais d'un localisme que l'on pourrait traiter de "constructiviste", au moins en partie: la langue ne sert pas uniquement à décrire le monde tel qu'il est, elle sert aussi à en évoquer une image subjective, à construire des représentations dans lesquelles entités et événements du monde peuvent être chargés de valeurs propres à l'interlocution.

Pour en revenir à l'analyse du verbe aller, Vandeloise traite ensuite la construction aller + infinitif, en commençant par les cas où aller garde un sens spatial (Yvonne va manger dans le sens où Yvonne se déplace pour manger). Il propose une règle très proche de la règle $R_{1}$ , entérinant ainsi la proximité sémantique de ces deux types d'emplois : $\mathrm{R}_{2}: x$ va $V_{\text {inf }}$ si $x$ change de lieu au moment de l'énonciation et si $x V_{f}$ au lieu final anticipé. ( $V_{f}$ étant une forme finie du verbe dont l'infinitif est $V_{\text {inf }}$ ).

Le sens de futur proche est alors considéré comme une extension du sens spatial, conformément à l'approche localiste. L'extension consiste à appliquer le schéma spatial au domaine temporel, suivant en cela Langacker (1987). On remplace le mouvement concret effectué par le sujet du verbe aller par un mouvement "abstrait", dans le domaine temporel, effectué par le locuteur entre le moment de l'énonciation et le moment où $x V_{f}$ a lieu. Nous ne discuterons pas plus avant ce point, dans la mesure où Vandeloise ne fait là que reprendre une approche classique des grammaires cognitives.

\section{La caractérisation énonciative}

Comme nous le disions en introduction, Vandeloise ne se satisfait pas complètement de l'approche localiste, ou, plus précisément, il juge nécessaire de la compléter par une caractérisation plus abstraite capable d'englober les sens spatiaux et non-spatiaux sans faire dériver les seconds des premiers. C'est à la recherche de cette caractérisation abstraite qu'il consacre l'essentiel de la suite de son article ${ }^{2}$. Il propose en fin de compte une nouvelle règle, plus générale, qu'il formule de la façon suivante :

Corela, HS-7 | 2010 

contexte d'énonciation. Il faudrait donc être plus précis dans la formulation. En fait, avec le verbe aller, on peut soutenir au contraire que le contexte d'énonciation reste très actif. Quand on dit Il va pleuvoir plutôt que Il pleuvra, on met l'accent sur le fait que la situation météorologique présente permet d'anticiper la pluie future; on crée un lien entre le présent et l'événement à venir. L'emploi de aller implique en effet que c'est dans le contexte d'énonciation que se trouvent réunies les conditions de réalisation de l'événement en question. C'est en ce sens que l'on doit comprendre l'appellation de futur "proche" qui est traditionnellement attribuée à cette valeur de aller, car, comme on le sait bien, il ne saurait s'agir de proximité purement temporelle (on peut très bien dire: le Soleil va s'éteindre dans cinq milliards d'années). D'ailleurs Vandeloise est conscient de cette propriété de aller puisqu'il écrit par ailleurs : «Utilisé au présent d'actualité, le verbe aller garde un pied dans le contexte d'énonciation immédiat (le déplacement) alors que le second s'en détache, en route vers le terme anticipé du déplacement » (p. 354). C'est cette idée qui manque manifestement dans la formulation de la règle $\mathrm{R}$.

ebjection concerne les contraintes que nous avions dégagées au début de cet article quand nous avions discuté du choix méthodologique de Vandeloise de restreindre son étude à certains emplois de aller : il fallait que la caractérisation proposée puisse être étendue d'abord aux emplois dits de verbe de manière de déplacement, et ensuite aux emplois non spatiaux autres que l'acception de futur proche. Or le moins que l'on puisse dire, c'est que la règle $\mathrm{R}$ se prête mal à ces extensions. On ne voit pas comment l'on pourrait faire intervenir de quelque façon que ce soit un affranchissement du contexte d'énonciation dans des énoncés tels que Il va vite et Il va à pied, et encore moins dans Yvan va bien, Ce chapeau lui va bien, et Il se laisse aller. Au contraire, dans tous ces emplois, c'est bien dans le contexte d'énonciation que l'on est entièrement plongé. Il y a donc là un sérieux problème qui remet en question la définition énonciative proposée par Vandeloise, quel que soit son attrait par ailleurs. 


\section{Un retour au localisme?}

29 Avec cet échec de l'approche énonciative, la question se pose de savoir s'il est possible de donner une caractérisation abstraite du verbe aller compatible avec tous ses emplois ou s'il faut renoncer à ce que nous avons appelé dans l'introduction le deuxième niveau de description que semblait postuler Vandeloise dans le cadre théorique qu'il avait esquissé. Nous n'aurons pas ici la prétention de répondre définitivement à cette question: cela réclamerait une étude beaucoup plus approfondie, et, rappelons-le, notre objectif dans cet article reste avant tout de discuter des propositions de Vandeloise.

On peut néanmoins ouvrir une piste, dont on peut d'ailleurs s'étonner qu'elle n'ait pas été exploitée plus avant par Vandeloise. Il s'agit de la notion d'anticipation, qu'il avait pourtant mise en évidence dès sa première étude de aller (Vandeloise 1987). Comme on vient de le voir, la valeur de futur proche de aller repose sur le fait que le contexte d'énonciation permet d'anticiper la réalisation de l'événement sur lequel porte aller. Il y a donc là un point commun entre les emplois spatiaux de déplacement (Yvan va à Prague et Yvan va manger au sens où Yvan se déplace pour manger) et les emplois de futur proche ( Yvan va manger au sens où Yvan est sur le point de manger). Dans tous les cas on constate qu'Yvan s'engage (ou est déjà engagé) dans un processus dont on peut anticiper qu'il conduira à la réalisation de Yvan être à Prague ou Yvan manger (que ce soit ailleurs ou sur place). Contrairement à la formulation de Vandeloise, celle-ci ne fait pas de aller le vecteur d'un affranchissement du contexte d'énonciation. Elle insiste plutôt sur le fait que le contexte présent est gros de l'événement futur. Cela permet du coup d'étendre beaucoup plus facilement la caractérisation aux emplois dits de manière de déplacement : en effet dans Yvan va vite ou Yvan va à pied,on indique aussi que Yvan est engagé dans un processus, sauf que cette fois on se contente de le qualifier au lieu de préciser quel en sera l'aboutissement anticipé.

On peut de même étendre assez facilement cette caractérisation à la plupart des emplois non-spatiaux de aller, mais à condition d'accepter une approche constructiviste plus radicale. Il faut supposer que le processus en question a lieu dans un espace qui est construit par la parole, que l'on peut appeler une scène verbale (Victorri 1999), et qui n'est pas forcément une représentation de l'espace physique. Formulée en termes de forme schématique (cf. Victorri 1999), cela donnerait la caractérisation suivante :

$\mathrm{FS}$ aller convoque un élément $\mathrm{E}$ de scène verbale (entité, procès, ou scène) et il évoque le fait que $\mathrm{E}$ est le siège d'une dynamique bien déterminée sur la scène verbale.

Cette caractérisation englobe tous les emplois spatiaux: dans ce cas, la scène verbale représente l'espace physique, et le fait que l'élément $\mathrm{E}$ (en l'occurrence une entité mobile) soit le siège d'une dynamique déterminée implique bien qu'il puisse y avoir, en présence d'un complément adéquat, anticipation de l'aboutissement du processus enclenché par cette dynamique (en l'occurrence un déplacement spatial finalisé). Elle englobe aussi les emplois de futur proche: dans ce cas, l'élément $\mathrm{E}$ est la scène toute entière, et c'est l'aboutissement anticipé de la dynamique dans laquelle est engagée cette scène qui est évoqué par la prédication sur laquelle porte aller. Comme on pourra le vérifier, cette formulation s'applique aussi bien aux constructions impersonnelles (Il va pleuvoir) qu'aux constructions personnelles (Yvan va manger), et, dans ce dernier cas, le sens de futur proche et bien distinct du sens de déplacement: le siège de la dynamique n'est pas de 
même nature dans les deux cas (une entité mobile pour le sens de déplacement et la scène tout entière pour le futur proche). Cela permet d'expliquer notamment pourquoi l'ambiguïté disparaît dans Yvan est allé manger, qui implique forcément un déplacement, et, plus généralement, pourquoi aller peut se construire avec l'auxiliaire être quand et uniquement quand il convoque une entité : c'est le fait que l'entité en question soit le siège $\mathrm{du}$ procès qui fait de aller un verbe en partie inaccusatif (l'opposition inaccusatif / inergatif devant être vue comme un continuum entre deux pôles plutôt qu'une dichotomie très tranchée).

Dans ce cadre, le traitement de la plupart des sens non-spatiaux ne présente pas de difficulté particulière. On n'a pas besoin de faire appel à un procédé métaphorique pour traiter Yvan va bien ou ça va bien: la manière de vivre ou de fonctionner sont, comme la manière de se déplacer, des qualifications d'une dynamique: c'est la nature de la dynamique qui change d'un exemple à l'autre (notons d'ailleurs que avoir de l'allant signifie précisément avoir du dynamisme). Des constructions comme se laisser aller ou aller sur ses quarante ans sont aussi assez bien couverts par cette forme schématique. Il n'y a guère que les emplois du type $C e$ chapeau va bien à Yvan qui restent problématiques: il semble en effet assez artificiel d'attribuer une dynamique particulière au chapeau en question... Peut-être pourrait-on considérer alors que c'est l'ensemble Yvan + ce chapeau qui est le siège de la dynamique (ce qui serait corroboré par l'inacceptabilité de la forme * Ce chapeau est bien allé à Yvan)?

Nous n'irons pas plus loin dans ces considérations : une fois de plus, notre objectif ici n'est pas de proposer une nouvelle analyse du verbe aller, mais simplement de montrer qu'il y avait là une voie qu'aurait pu explorer Vandeloise pour le niveau de description plus abstrait qu'il recherchait. En fait, là où Vandeloise recherchait à échapper au localisme en adoptant une approche intentionnelle ou énonciative, nous proposons au contraire de garder l'outillage topologico-dynamique hérité de l'espace physique, mais en l'appliquant à ce que nous appelons des scènes verbales, c'est-à-dire des sortes d'espaces mentaux construits par la parole, de statut phénoménologique bien particulier (cf. Victorri 1999). Ainsi, dans l'approche que nous suggérons, aller resterait fondamentalement un verbe "spatial", au sens où il évoquerait bien un mouvement dans un espace. Mais cet espace n'étant plus l'espace physique, même enrichi comme le concevait Vandeloise, on ne peut plus vraiment parler de localisme, à moins de jouer sur les mots.

\section{La genèse du langage}

Un dernier aspect de l'article de Vandeloise que nous voudrions discuter concerne ses réflexions sur la genèse du langage. Même si elles ne sont pas très développées dans le cadre de cet article, elles sont intéressantes parce qu'elles touchent un problème-clé de l'origine du langage, sur lequel les linguistes ont beaucoup à apporter dans cette grande entreprise multidisciplinaire qu'est devenue la recherche dans ce domaine. Ce problème, c'est de comprendre pourquoi et comment le langage s'est-il autant complexifié. En effet, dans la plupart des scénarios qui nous sont proposés aujourd'hui, on ne voit pas pourquoi se sont développées un certain nombre de caractéristiques des langues qui en font des systèmes très spécifiques, et bien plus complexes que les autres systèmes de communication animale connus : la récursivité syntaxique bien sûr, mais aussi nombre de particularités grammaticales comme les systèmes de marqueurs modaux et aspectuels, ou 
encore des propriétés lexicales comme la polysémie. Sur ces questions, la contribution des linguistes est absolument essentielle, dans la mesure où seule l'analyse fine du fonctionnement et de la structure des langues permet d'avancer des hypothèses consistantes avec la réalité du langage. Par exemple, en ce qui concerne la récursivité, on peut citer le travail de Le Goffic (2001) qui propose un processus de genèse de la subordination permettant de rendre compte de la mise en place de la récursivité syntaxique (cf. Victorri 2007).

La contribution de Vandeloise, elle, porte sur le passage d'un système de communication centré sur la situation de communication (le ici et le maintenant) à un système capable de s'en détacher pour remplir de nouvelles fonctions, et notamment la fonction narrative, dont on peut supposer qu'elle a joué un rôle capital dans la complexification du langage (cf. Victorri 2002).

37 Vandeloise fait l'hypothèse d'une complexification progressive des énoncés primitifs (qu'il appelle des "actes linguistiques") par l'ajout de constituants : on passerait ainsi d'actes linguistiques à un constituant à des actes linguistiques à deux puis trois puis quatre constituants. On peut rapprocher ce processus des deux premières étapes du scénario de Jackendoff (1999) qui propose de distinguer sept étapes dans la complexification du langage, avec des énoncés à un seul symbole dans la première étape, puis la concaténation de plusieurs symboles dans la deuxième. Le résultat, dans les deux cas, c'est un système qui ressemble à ce que Bickerton a appelé un protolangage (Bickerton 1990), très efficace effectivement pour communiquer sur la situation d'interlocution, mais encore déficient pour évoquer d'autres contextes que le contexte d'énonciation immédiat. La proposition de Vandeloise est alors qu'un verbe comme aller, c'est-à-dire un verbe capable de décrire un déplacement finalisé, aurait pu être le premier support de l'évocation de scènes hors du contexte d'énonciation immédiat. Comme il l'écrit joliment: «Un jour, le langage s'est arraché à la référence et c'est dans le verbe aller qu'il a trouvé ses ailes » (p 354).

Cette idée d'une complexification du protolangage par des marques grammaticales du futur (ou plutôt du prospectif) issues d'unités lexicales servant à évoquer le déplacement est tout à fait intéressante. On pourrait d'ailleurs émettre des hypothèses analogues pour l'apparition d'autres marques grammaticales, comme les marques du passé. En somme l'idée est que les processus de grammaticalisation auraient commencé dès le protolangage, bien avant donc que des langues à part entière existent et puissent, elles aussi, être le siège de ces processus au cours de leur évolution. On conçoit alors pourquoi Vandeloise a été séduit par une caractérisation du verbe aller en terme de contextes d'énonciation (la règle $\mathrm{R}$ ) : cela correspondait bien à l'idée qu'il se faisait d'un deuxième niveau de description, plus abstrait, qui devait, dans le cadre théorique qu'il était en train de construire, révéler les structures fondamentales du langage. Comme il l'écrit dans sa conclusion: "la règle $\mathrm{R}$ s'inscrit dans une genèse idéale de la langue qui cherche à distinguer les étapes cruciales sur le chemin qui conduit des premiers actes linguistiques au langage que nous connaissons » (p. 358). C'est visiblement cette " genèse idéale » (qu'il appelle aussi "genèse logique du langage » p.345) qui était devenue l'un des objets essentiels de ses réflexions, et qu'il aurait aimé pouvoir continuer à déchiffrer et à comprendre. 


\section{BIBLIOGRAPHIE}

Bickerton D. (1990) : Language and Species, University of Chicago Press.

Cadiot P., Lebas F., Visetti Y.-M. (2006) : The semantics of the motion verbs: action, space, and qualia, in M. Hickman, S. Robert (éds.), Space in language: linguistic systems and cognitive categories, John Benjamins, p. 175-206.

Fauconnier G. (1985) : Espaces mentaux,Editions de Minuit.

Fauconnier G. (1997) : Mappings in thought and language,Cambridge University Press.

François J. (1992) : La causation et l'action dans la théorie sémantique de Ray Jackendoff, Intellectica, 13-14, p. 291-326.

Jackendoff R. (1999) : Possible stages in the evolution of the language capacity, Trends in Cognitive Sciences, 3:7, p. 272-279.

Lakoff G., Johnson M. (1980) : Metaphors We Live By, University of Chicago Press.

Langacker, R. (1987) : Mouvement abstrait, Langue française,76, p. 59-76

Lyons J. (1980) : Sémantique linguistique, Larousse.

Le Goffic P. (2001) : Sur les sources et le développement de la subordination dans le langage : l'exemple de l'indo-européen, Recherches en Linguistique et Psychologie cognitive, 16, Presses universitaires de Reims, p. 25-56.

Slobin D. I. (2003) : The many ways to search for a frog: Linguistic typology and the expression of motion events, in S. Strömqvist and L. Verhoeven (éds.), Relating Events in Narrative: Vol. 2 , Lawrence Erlbaum, p. 219-257.

Talmy L. (1985) : Lexicalization patterns: Semantic structure in lexical forms, in T. Shopen (éd.), Language typology and syntactic description: Vol. 3, Cambridge University Press, p. 36-149.

Vandeloise C. (1986) : L’espace en français : sémantique des prépositions spatiales, le Seuil.

Vandeloise C. (1987) : La préposition à et le principe d'anticipation, Langue française,76, p. 77-111.

Vandeloise C. (2005) : Perspectives spatiales et relativité linguistique, in A.M. Russo, S. Harel (éds.), Lieux propices: l'énonciation des lieux, le lieu de l'énonciation dans les dans les contextes francophones interculturels, Presses de l'Université de Laval, p. 295-310.

Vandeloise C. (2006) : Are there spatial prepositions? , in M. Hickmann, S. Robert (éds.), Space in languages: linguistic systems and cognitive categories, John Benjamins, p. 139-154.

Vandeloise C. (2007) : Le verbe ALLER : L'affranchissement du contexte d'énonciation immédiat, Journal of French Language Studies, 17:3, p. 343-359.

Victorri B. (1999) : Le sens grammatical, Langages, 136, p. 85-105.

Victorri B. (2002) : Homo narrans : le rôle de la narration dans l'émergence du langage, Langages, 146, p. 112-125.

Victorri B. (2007) : Termes en kw-, récursivité et origine du langage, in M. Charolles, N. Fournier, C. Fuchs, F. Lefeuvre (éds.), Parcours de la phrase, Ophrys, p. 259-273. 


\section{NOTES}

1. Cité par Jacques François (1992, p. 292).

2. Dans une version préliminaire de cet article (Vandeloise, communication personnelle), il explore d'abord la piste, apriori séduisante, de l'intentionnalité, en énonçant la règle : R3 : $\mathbf{x}$ va (Prep) y si l'intention de $\mathbf{x}$ est (être Prep) y. Cette définition a bien l'avantage de traiter aussi bien les énoncés du type Yvan va à Paris que ceux du type Louis va travailler, quel que soit le sens retenu pour ces derniers (déplacement ou futur proche). Mais malheureusement aller peut exprimer un futur proche même quand l'action n'est pas intentionnelle: comme le fait remarquer Vandeloise à propos de l'énoncé Louis va échouer, il est peu probable que l'intention de Louis soit d'échouer. On peut ajouter que aller exprime aussi le futur proche dans des constructions impersonnelles (Il va pleuvoir), donc en l'absence de tout sujet agentif à qui prêter une intention.

\section{RÉSUMÉS}

L'un de ses derniers articles de Claude Vandeloise est consacré à l'analyse du verbe français aller. Il en donne deux caractérisations : l'une peut être traitée de localiste au sens large puisqu'elle fait appel à l'espace doté de propriétés fonctionnelles et intentionnelles, tandis que l'autre est plus originale dans son cadre théorique, puisqu'elle repose sur la notion de contexte d'énonciation. Il s'agit donc d'une évolution assez importante dans la pensée de Vandeloise que nous essayons de décrypter ici.

In one of his last papers, Claude Vandeloise analyses the French verb aller. He gives two characterizations of this verb. One of them is "localist" in a broad sense, since it uses spatial notions enriched with functional and intentional features. The other one is more original in his theoretical framework, since it is based on the notion of enunciation context. This is a rather important evolution in Vandeloise's conceptions that is deciphered here.

\section{AUTEUR}

\section{BERNARD VICTORRI}

LaTTiCe, UMR 8094, CNRS-Ecole Normale Supérieure 